\title{
A FAILURE INDEX FOR HPC APPLICATIONS
}

\author{
Andrei Păun ${ }^{\mathrm{a}, \mathrm{b}, *}$, Clayton Chandler ${ }^{\mathrm{c}}$, Chokchai Leangsuksun ${ }^{\mathrm{c}}$, Mihaela Păun ${ }^{\mathrm{c}, \mathrm{d}}$ \\ ${ }^{a}$ ICUB/Faculty of Mathematics and Computer Science, University of Bucharest, Bucharest 010014, \\ Romania \\ ${ }^{b}$ Escuela Técnica Superior de Ingenieros Informáticos, Universitad Politécnica de Madrid, Madrid \\ 28660, Spain \\ ${ }^{c}$ College of Engineering and Science, Louisiana Tech University, Ruston, LA, 71272, USA \\ ${ }^{d}$ Bioinformatics Department, National Institute for R\&D for Biological Sciences, Bucharest 060031, \\ Romania
}

\begin{abstract}
This paper conducts an examination of log files originating from High Performance Computing (HPC) applications with known reliability problems. The results of this study further the maturation and adoption of meaningful metrics representing HPC system and application failure characteristics. Quantifiable metrics representing the reliability of HPC applications are foundational for building an application resilience methodology critical in the realization of exascale supercomputing. In this examination statistical inequality methods originating from the study of economics are applied to health and status information contained in HPC application log files. The main result is the derivation of a new failure index metric for HPC - a normalized representation of parallel application volatility and/or resiliency to complement existing reliability metrics such as mean time between failure (MTBF), which aims for a better presentation of HPC application resilience. This paper provides an introduction to a Failure Index (FI) for HPC reliability and takes the reader through a use-case wherein the FI is used to expose various run-time fluctuations in the failure rate of applications running on a collection of HPC platforms.
\end{abstract}

Keywords: Failure Index (FI), inequality measures, High Performance Computing, resilience, system volatility, adequate level of performance

\section{INTRODUCTION}

The provision of resilient HPC applications running on innately unreliable hardware is a grand challenge within the exascale supercomputing community. Such development effort requires the coordination amongst computer scientists, mathematicians, application developers, production system operators and domain experts from various government

\footnotetext{
* Corresponding author.

Email addresses: apaun@fi.upm.es, apaun@fmi.unibuc.ro (Andrei Păun), cfchandler@gmail.com (Clayton Chandler), box@coes.latech.edu (Chokchai Leangsuksun), mihaela.paun@incdsb.ro (Mihaela Păun) 
laboratories, private organizations and academic institutions. One of key enablers of such collaboration is the maturation of a consistent ontology for the metrics and data utilized within the HPC research and development community. This community must both re-examine the utility of existing metrics in anticipation of exascale's failure-rich computing environments as well as develop new statistics appropriate to the study of HPC application resilience in this regime [7], [12], [17], [23], [24], [25]. The availability and performance of systems based on different reliability metrics have been considered in the literature in the last decade in various studies [10], [13], [16], [20]. This paper suggests a new metric: the Failure Index (FI) as an interplay of various reliability indices and relates to the Gini and Atkinson indices from Econometrics. Other authors have previously considered the Gini/econometric indices in the area of HPC, for example in [4], [14] and [15] Gini is used for making better scheduling decisions at the level of cloud services or to model the aging of systems, but no other authors so far have used Atkinson or more sophisticated constructs for gathering insight in subsets of the system.

We use the FI index to study failure-rich HPC application log files from Los Alamos Laboratory, data described in [6], [19] and also in [23]. Prevously the data has been used differently from our approach: in [19] the authors are studying the data from a data mining and clustering perspective while in [23] the data is analyzed with basic statistical tools: mean, median, using the cumulative distribution function (CDF) to determine the distribution of failures, etc. The FI exposes fluctuations in the failure rate of an HPC system it operates. HPC platforms with substantial fluctuations in failure activity are defined to be more volatile than those with more consistent failure modes. Importantly, the FI is not intended to replace MTBF or related metrics as a measure of system availability. Rather, the FI should be used as a complement to these measures in expressing HPC failure modes, giving administrators and developers a more robust view of the health and status of their operational HPC environment.

The Gini index is a measure of dispersion frequently used to study inequality, which indicates total inequality in a sample. Both the traditional measures of central tendency, as well as the inequality indices (Gini, Atkinson, etc.) incorporate value judgment, hence not one of them should be considered as the complete measure. While using the measures such as mean time to failure (MTTF), tends to ignore extreme or outlier values, if the two extremes cancel each other and do not change the value of the mean (as compared to a sample that has values around the mean), the Gini index is very sensitive to outliers, even in large samples.

\begin{tabular}{|l|l|l|l|}
\hline Nodes Reliability N=10 & Scenario 1(100, 80, & Scenario 2(212, 75, & Scenario 3(246, 66, \\
& $84,67,93,98,77,72$, & $81,61,57,120,72$, & $68,68,68,68,68,68$, \\
& $91,94)$ & $60,60,58)$ & $68,68)$ \\
\hline Mean & 85.6 & 85.6 & 85.6 \\
Median & 87.5 & 66.5 & 68 \\
Coefficient of Variation & 13.23 & 56.39 & 56.37 \\
Gini & 0.07 & 0.23 & 0.19 \\
Atkinson $(\epsilon=0.5)$ & 0.004 & 0.051 & 0.058 \\
\hline
\end{tabular}

Table 1: Scenarios for expressing inequality measures.

In Table 1, we can see why the measures of inequality are better used complementarily, rather than using them as a single approach. One can see that the mean has the same 
value for the samples in the three scenarios, hence we could not identify if there is any difference between the scenarios. The coefficient of variation of Scenario 1, identifies the fact that there is less variation among the nodes reliability, when compared to scenarios 2 and 3. Fact supported also by the value of the Gini index, which is smaller and closer to 0 when measuring the inequality in reliability for Scenario 1 . However, when comparing Scenarios 2 and 3, we obtain the same mean, and an almost identical coefficient of variation. However, even by visual inspection of the sample, one cannot say that the two scenarios exhibit the same reliability characteristics. Inspecting the values of the Gini index, we can say that there is less inequality in the node's reliability for Scenario 3, as it is for Scenario 2. This is due to the fact that Gini investigates the global inequality in a system, however does not capture where in the distribution does the inequality occur. Consequently, we can have two very different distributions of failure with the same Gini index values.

On the other hand, Atkinson index offers the possibility to examine inequality in different areas of the failure distribution, and this is where it overcomes the Gini's index inability.

We have used in the above example the parameter 0.5 , basically looking at how the inequality looks like in the upper part of the distribution, meaning that we look at the long term failures. Overall there is less inequality in the Scenario 3 (fact captured by the Gini index), but there is more inequality captured in the upper failures (captured by the Atkinson index).

However, Atkinson's index is not enough by itself since it is much more sensitive to the lower part of the distribution (due to its nature: defined for poverty analysis). Due to this fact we need to define another index that is inspired by Atkinson's but it is designed for the failures of HPC systems. We call the newly defined index the Failure Index (FI).

Given the innately unreliable nature of exascale hardware platforms, we theorize that systems with lower FI's - those with more predictable failure modes - present better opportunities for preemptive error handling or fault prediction techniques, even if the severity of those individual failures are greater than those occurring in the more volatile environment.

We believe that by considering an ensemble of the FI and related inequality indices (such Gini and Atkinson indices) one would better highlight outliers (nodes) and their reliability characteristics in a way that will exhibit a clearer picture of the true resilience or reliability of such large scale systems.

Data required to compute the FI could be periodically pulled from the system using instrumentation tools and stored in the system's file system. The FI and associated statistics could be continuously updated and monitored to provide a near-real time view of application reliability trend to the scheduler, other management tools or the application itself.

For example, if one were to construct a reliability-aware metascheduler tasked with processing incoming jobs and scheduling those jobs to a farm of various heterogeneous computing resources, knowing the FI coefficients for each application/system permutation would yield more intelligent scheduling decisions. These metrics can be combined with additional information pertinent to the power footprint and raw failure rate of each permutation to maximize data center efficiency. The failure index of a given permutation can assist finding the appropriate trade space within the triangular computing constraints of performance, power and reliability. 
The paper is structured as follows: the next part, Section 2 defines formally inequality indices and describes their previous application to HPC failure. Section 3 describes the proposed Failure Index and Section 4 gives the results of the paper applying the newly defined index on real data, the section finishes with discussions and comments. The conclusion and future work are discussed in Section 5 .

\section{INEQUALITY METRICS AND THEIR APPLICATION TO HPC FAIL- URE DATA}

\subsection{INEQUALITY METRICS}

The indices that prompted the creation of the Failure Index are well known econometric measures/metrics such as the Gini and Atkinson indices as well as the Lorenz curve. More specifically the idea of the new index defined in this paper is based on a modified version of the Gini index used to analyze aging or rejuvenating objects, which was defined in [14]. While these indices have been widely applied in areas outside of econometrics, it is a novel idea to apply them in the context of HPC application reliability. Inequality indices are based on the Lorenz curve. This curve, introduced in 1905 by Max Otto Lorenz as a graphical representation of a distribution's level of equality, compares observed events with distributions featuring perfect equality [18]. The Lorenz curve is based on a convex function and has been widely adopted by economists for use in comparing income distributions.

If the Lorenz curve is represented by the function $Y=L(X)$, where $X$ is the population percentile defined by income, the Gini index can be formalized as

$$
G=1-2 \int_{0}^{1} L(x) d x
$$

From the definition it is easy to show that the Gini index has values between 0 and 1. Both the Lorenz curve and Gini index can be applied to either continuous or discrete distributions. The Atkinson index is a normalized measure of the statistical inequality found in a discrete data set [1]. An Atkinson value of 0 indicates total equality while a value of 1 indicates total inequality. The Atkinson index is defined using the formula:

$$
A_{\epsilon}\left(y_{1}, \ldots, y_{n}\right)= \begin{cases}1-\frac{1}{\bar{y}}\left(\frac{1}{N} \sum_{i=1}^{N} y_{i}^{1-\epsilon}\right)^{\frac{1}{1-\epsilon}} & , \epsilon \neq 1 \\ 1-\frac{1}{\bar{y}}\left(\frac{1}{N} \prod_{i=1}^{N} y_{i}\right)^{\frac{1}{N}} & , \epsilon=1\end{cases}
$$

$\bar{y}$ is the arithmetic mean of the observations $y_{1}, \ldots, y_{n}$. The parameter $\epsilon$ is called the sensitivity parameter, which has usually a value between 0 and 1 . An $\epsilon$ approaching 0 represents an Atkinson index more sensitive to changes in the upper end of the distribution and an $\epsilon$ approaching 1 represents an Atkinson index more sensitive to changes in the lower end of the distribution. For all fixed single data sets $y_{1}, \ldots, y_{n}$ that we have considered the Atkinson index $A_{\epsilon}\left(y_{1}, \ldots, y_{n}\right)$ is an increasing function of $\epsilon$ wherein larger values of $\epsilon$ lead to larger indices. For more information and details we refer the interested reader to $[2]$. 


\subsection{THE GT INDEX}

This section introduces the GT index first proposed by [14], which was developed based on the Gini index for use in the evaluation of systems with repairable parts (for which an HPC application or system certainly qualifies - HPC system nodes can be replaced and HPC application processes can be rebooted).

When utilizing the GT index, time serves as the independent variable. The GT index models the distribution in question as a Poisson process (PP) and measures that distribution's trend toward and away from a heterogeneous Poisson process (HPP) in time. The GT index is defined as

$$
G T(N(T))=1-\frac{2 \int_{0}^{T} N(t) d t}{T \times N(T)}
$$

$N(T)$ represents the number of failure events occurring in the given time interval $[0, T]$

The GT index returns values ranging from -1 to 1 . A system with a decreasing rate of occurence of failures (ROCOF) results in indices less than 0. Increasing ROCOF results in values greater than 0 . Evaluating the ROCOF at time $t$ is a different approach for evaluating reliability than the Atkinson index; ROCOF represents the absolute rate at which system failures occur. Systems with ROCOF values closer to 1 or -1 improve or fail more rapidly than those with values closer to 0 . The $G T$ index allows us to examine changes in failure rate with respect to time and, when combined with $F I$, creates a more robust understanding of the failure behavior of a given system.

\section{THE FAILURE INDEX}

The Failure Index which is the main result of this paper is described and defined formally in this section. The FI is based on the Atkinson index, the Lorenz curve and ROCOF. It is constructed as a time-dependent implementation of the Atkinson index, therefore also containing a sensitivity parameter that allows investigators to place emphasis on various segments of a distribution. It is important to note that an FI is not a measure of good system behavior. Long stretches of non-failure activity have just as much impact on the resulting index as equally long stretches of continuous and regular failure activity. The FI only captures these fluctuations and does not distinguish between these cases, thus it is best applied as part of an ensemble of statistics. One can note that, FI indices from instrumented HPC performance data could be used to design and execute dynamic scheduling for HPC workflows, as in [4].

One can conclude that the time between successive failures and the time required to recover from the failure significantly contribute to the overall failure volatility exhibited by an HPC system. The time between failures is not accounted for by traditional inequality indices such as the Atkinson index. The ROCOF considers the time between failures but not the time it takes to recover from the failure. The FI, however, captures the impact of both the time between failures and the recovery time. Results show that a system with more regular time intervals between system failures and similar recovery times exhibit lower levels of volatility than those with prolonged periods of highly unequal time between successive failures and those with highly unequal recovery times. FI 
values are less affected by single large failures than Atkinson index values, though the level of inequality in the impact of each failure exhibited by the system does still play a role in the volatility reported by the FI. One should also notice that, for the sensitivity parameter $\epsilon=0$, the FI is essentially a ROCOF for failure impact, rather than for the number of failures exhibited (also see Table 2).

\begin{tabular}{|l|l|}
\hline$F I \geq \frac{1}{2}$ & $\begin{array}{l}\text { Indicates that the system is becoming less volatile. The impact of recent } \\
\text { failures is not as great as the impact of failures earlier in the system's lifetime. }\end{array}$ \\
\hline$F I<\frac{1}{2}$ & $\begin{array}{l}\text { Indicates that the system is becoming more volatile. That is, the impact of } \\
\text { recent failures is greater than that of earlier ones. }\end{array}$ \\
\hline
\end{tabular}

Table 2: General characteristics of the Failure Index.

The GT index [14] allows one to examine changes in failure rate with respect to time, which, when combined with FI, will better describe the failure behavior of a given system over time. We show that the FI and Atkinson indices complement existing measures in the HPC environment, such as ROCOF and the GT index. The differences and similarities of the indices discussed above are summarized in Table 3.

\begin{tabular}{|l|l|l|l|l|}
\hline Index & Atkinson & GT & ROCOF & FI \\
\hline Type of data used & $\begin{array}{l}\text { Discrete, order } \\
\text { does not mat- } \\
\text { ter }\end{array}$ & $\begin{array}{l}\text { Continuous } \\
\text { data, order } \\
\text { of occurrence } \\
\text { matters }\end{array}$ & $\begin{array}{l}\text { Continuous } \\
\text { data, order } \\
\text { of } \begin{array}{l}\text { Occurrence } \\
\text { matters }\end{array}\end{array}$ & $\begin{array}{l}\text { data, } \\
\text { of } \\
\text { matters }\end{array}$ \\
\hline $\begin{array}{l}\text { Consider different fail- } \\
\text { ore sources }\end{array}$ & Yes & No & No \\
\hline Sensitivity parameter & Yes & No & No & Yes \\
\hline $\begin{array}{l}\text { Accounts for time be- } \\
\text { tween failure }\end{array}$ & No & Nos & No & Yes \\
\hline $\begin{array}{l}\text { Accounts for recovery } \\
\text { time from failure }\end{array}$ & Yes & Yes & \\
\hline
\end{tabular}

Table 3: Summary of the metrics' properties.

Fluctuations between various system failure modes are not captured by existing metrics such as MTBF. As is the case with the use of different inequality metrics in econometrics, we do not advocate for the application of the FI as a one-size-fits-all replacement for current metrics used in HPC failure analysis. The FI is a complementary metric that can be used to provide a novel look into aspects of system behavior not previously captured.

The occurrence of failures is often modeled as a Poisson process. When considering the FI, this is slightly modified. We do not count discrete events, but rather use the amount of resulting downtime as the impact exhibited by the failure. Hence the analysis of failure impact could be modeled with the compound Poisson process (well known in probability theory), which is a continuous-time (random) stochastic process with jumps. The jumps arrive randomly according to a Poisson process and the size of the jumps is also random, with a specified probability distribution.

We derive the Failure Index $(F I)$ by combining concepts of the Atkinson index, the Lorenz curve and ROCOF. The $F I$ is mathematically defined as 


$$
F I_{\epsilon}(F(T))=1-\frac{1}{F(T)}\left(\frac{1}{T} \int_{0}^{T} F(t)^{1-\epsilon} d t\right)^{\frac{1}{1-\epsilon}}
$$

$F(t)$ represents the CDF of the system downtime resulting from failure, and $F(T)$ represents the cumulative system downtime at a given time $T$. The $F I$ is constructed as a time-dependent implementation of the Atkinson index. Like the Atkinson, it contains $\epsilon$ , $0 \leq \epsilon \leq 1$ which allows the $F I$ to place emphasis on various segments of a distribution. Parameters closer to (but not equal to) 1 generate $F I^{\prime} s$ that give greater weight to the larger elements of the distribution than parameters closer to 0 . The $F I$ represents the level of volatility in the impact of failures encountered by a given system at time $T$ using a given parameter. Unlike the $G T$ index, the $F I$ addresses not only the number of failures, but the resulting downtime as well. The limiting values of the parameter $\epsilon$, represent either indifference regarding the nature of the distribution $(\epsilon=0)$ or concern only with values found in the lowest downtime group. The choice of $\epsilon$ depends on the investigator and the nature of the data. After a certain value of $\epsilon$, which we (and the literature for the Atkinson index) consider to be 0.2 , only the lowest downtime group is emphasized. As $\epsilon$ approaches 1, the index becomes more sensitive to the upper end of the downtime distribution. When $\epsilon=1$, the index is 0 , indicating that one failure causes all downtime found in the data set. Note that, because $F(T)$ can be expected to be zero for small values of time $T$, values $\epsilon>1$ are not feasible in this analysis.

It is important to note the difference between the information captured by inequality metrics such as Atkinson indices and the information captured by the FI. The Atkinson index is calculated with respect to a series of discrete, weighted events. The FI is calculated with respect to cumulative time-dependent data. Atkinson indices capture the level of inequality amongst multiple individual failure events, whereas the FI captures the overall level of volatility exhibited by the system in time.

The function $F(t)$ that records the cumulative system downtime quantified within the $F I$ is similar to the function used in ROCOF that captures the number of failures. This means that for $\epsilon=0$ the $F I$ equates to a ROCOF for failure impact or the rate of changes, rather than for the number of failures, which is scaled on the interval $[0,1]$ rather than the interval $[-1,1]$. Consequently, the interpretation is similar. For $\epsilon=0$, an $F I>0.5$ (the $F I$ for $F(t)=t$, the line of zero volatility) indicates that the system is becoming less volatile and the impact of recent failures is not as great as the impact of failures earlier in the system's lifetime. Note that the number of failures may still be increasing because the system could change from having few catastrophic failures to having many easily repaired ones. Conversely, for $\epsilon=0$, an $F I<0.5$ indicates that the system is becoming more volatile and the impact of recent failures is greater than that of earlier ones. Again, note that the number of failures may actually be decreasing as many earlier, easily fixed failures earlier may now be replaced with a few catastrophic ones.

In the above fashion the FI complements information obtained from other parameters such as ROCOF and existing reliability measures such as MTBF. The occurrence of failures is often modeled as a Poisson process, thus the analysis of failure impact could be modeled with Poisson processes in which the time interval is not unit. Such processes are called compound Poisson process and have been investigated in the literature [21], [3]. 
The sensitivity parameter $\epsilon$ allows us to scale the numbers, but the effect is rather subtle. First of all, an easy integration shows that the $F I$ for $F(t)=t$, which we call the line of zero volatility, is $1-\left(\frac{1}{2-\epsilon}\right)^{\frac{1}{1-\epsilon}}$.

This number ranges from 0.5 for $\epsilon=0$ to $1-e^{-1}$ as $\epsilon$ approaches 1 . For small values of $\epsilon$, the behavior of the $F I$ is similar to that for $\epsilon=0$. For $\epsilon$ close to 1 , the $F I$ typically returns numbers greater than when behavior is volatile. That is, for $\epsilon$ close to 1 , the FI indicates that there is volatility, but it does not indicate when the volatility occurs. Here we see that, in some situations, the parameter $\epsilon$ helps extract not just information complementary to the ROCOF, but more information than possible with the ROCOF. For example, consider a system that halfway through the observation window experiences multiple failures, but few or no failures at the beginning or toward the end of the observation. The most extreme scenario would be exactly one failure halfway through the observation time. In this scenario, the ROCOF of the system would be 0 , indicating a system that neither heals, nor deteriorates. The actual evaluation of the system depends on other data. If the observation time was short and the downtime was large, then the system certainly is not healing. If the observation time was long and the downtime was small, then the system is not just healing, but actually healthy. For the same function the $F I$ is $1-\left(\frac{1}{2}\right)^{\frac{1}{1-\epsilon}}$, which with is close to 1 . That is, whereas the ROCOF gives inconclusive or neutral information, the FI alerts the investigator that deeper analysis may be needed. Using the terminology native to the LANL data set, existing inequality indices such as the Atkinson index measure the level of inequality contained in the downtime column. The FI measures the level of volatility in the total downtime column with respect to the total time perhaps in production. And the ROCOF measure failure impacts. Thus together are better representing system reliability than a traditional reliability measurement such as MTBF.

In this analysis FIs with different sensitivity parameters were generated for all 23 systems contained in the LANL data set. Similar to existing inequality indices such as the Atkinson index, the FI produces scale-and location-invariant indices. This allows for comparison of various machines regardless of their size. The FI is less sensitive to downtime caused by a single abnormally strong or weak failure and more sensitive to stretches of volatile or non-volatile failure behavior in time, as we will now demonstrate.

\begin{tabular}{|l|l|l|l|l|}
\hline System & FI $(\epsilon=0)$ & FI $(\epsilon=0.20)$ & FI $(\epsilon=0.99)$ & GT $(\mathrm{N}(\mathrm{T})))$ \\
\hline 19 & 0.4978905 & 0.5130915 & 0.6412173 & -0.02191605 \\
\hline
\end{tabular}

Table 4: Failure Indexes and ROCOF, System 19, LANL Data.

The data set displayed in Table 4 generates the $F I(\epsilon=0.99)$ for the above data set is 0.6412173 , which represents a substantial amount of failure volatility in the given time frame.

\section{RESULTS AND DISCUSSION}

We give in this section the results of applying the proposed index on real data. 


\subsection{DATA AND METHODOLOGY}

The data used in this study originates from 23 HPC machines housed at Los Alamos National Laboratory from 1996 to 2005. The data set containing this information was publicly released in 2005 and initially analyzed in [23]; one can see more details about the available data and examples in [6] and [19]. This data set was chosen for our work due to its scale and heterogeneity. Covering 23 different systems and months of data, these $\log$ files demonstrate the scale- and location-invariant nature of inequality indices. This data set is one of the few made publicly available representing HPC behavior at a large scale and it has been analyzed from various points of view in the literature. The data set contains 23,739 failure events from 23 of LANL's systems, accrued over a nine-year period. To maintain confidentiality, each system is labeled using a number system from 2 to 24 (Systems 2, 3, etc.), with System 2 being the oldest system recorded in the log and System 24 being the youngest. Each record contains a time stamp of when a failure was first reported (labeled prob started), the time when the issue causing the failure was resolved (prob fixed), the total system downtime (in minutes) resulting from the failure, the specific system and node affected by the failure, the type of workload running on the node at the time of the failure and the root cause of the failure. Failures are detected by an automated monitoring system that pages operations staff whenever a node goes down. The operations staff then creates a record in a database specifying the time of the failure and the system and node affected. They then turn the node over to a system administrator for repair. Upon repair, the system administrator notifies the operations staff, which then puts the node back into the job mix and fills in the end time of the failure record. If the system administrator was able to identify the root cause of the problem, he provides operations staff with the appropriate information for the root cause field of the failure record. Otherwise, the root cause is specified as undetermined. The data set covers each of the 23 systems only during their time in a production environment; time allotted for testing or maintenance is not covered.

Although covering multiple systems, the LANL data set is similar to previously released failure logs from Lawrence Livermore National Laboratory's Blue Gene/L (BG/L) system [11] in that source of failure are broken up into facilities. Facilities are components of the system that potentially serve as points of origination for failure. The facilities themselves are, however, not identical to those found in the LLNL data set. Instead of the ten facilities represented in the LLNL data, the LANL dat aset only contains five: HARDWARE, SOFTWARE, NETWORK, HUMAN ERROR, and UNDETERMINED. Further, instead of the machine or operating system autonomously generating the facility of origin in the log file such as in the LLNL data set, LANL facilities are the result of human interaction. Operators record the suspected source of an error in a written log, which is later investigated and confirmed or changed at subsequent staff meetings. The LANL data also contains a column for noting whether the current failure event is due to a previously listed event, again the result of human interaction.

Columns in the LANL records represent the name (2, 3, etc.) and type (cluster, graphics card, etc.) of each system containing a logged event. Additional columns represent the number of nodes housed in the associated system, the total number of processes running on the machine at the time of the failure, as well as the number of processes running on each node in the machine at the time of the failure. Also recorded are the specific node reporting the failure, the installation date and production commission and 
decommission dates of that node, the purpose of that node, the start and end dates of the failure in question, the total downtime in minutes caused by the failure in question, and finally the origin of that failure.

Our initial analysis pays specific attention to the system and downtime columns of each entry. Gini and Atkinson indices are generated for the failure behavior exhibited by each of the 23 machines found in the file. All of these failures are caused by one of the five possible facilities reported in the log. The scale- and location-invariant nature of inequality indices allows our analysis to compare entries reported by multiple machines with one another regardless of each system's node count or system type, which led to the selection of the LANL data set and its 23. We begin our analysis with the Atkinson index. Because the input for this analysis is data associated with various causes of failures, this section specifically examines inequalities among causes. Atkinson indices were calculated using two sensitivity parameters, $\epsilon=0.99$ and $\epsilon=0.20$. These indices were constructed with respect to the downtime caused by each failure per system recorded in the log file. Atkinson indices were generated for the $20^{t h}, 40^{t h}, 60^{t h}, 80^{t h}$, and $100^{\text {th }}$ percentile of events found in the LANL data set relative to each of the 23 systems. Each of the system's failures were grouped according to their time of occurrence with the first event in each group being the one generated by the earliest failure and the last event being the one generated by the latest failure. The percentiles represent the lifetime of each system based on the discrete data found in the log files. The resulting indices illustrate the level of inequality in downtime resulting from failures incurred by the systems. The resulting Atkinson $(\epsilon=0.20)$, and Atkinson $(\epsilon=0.99)$ indices were grouped according to percentile and averaged. Resultant values, shown in Table 5 , represent fluctuations in the impact or downtime caused by all failures across all systems in the data set. These results show that as these systems age they exhibit increased inequality in the amount of downtime resulting from failures. This suggests that as HPC systems age their failure activity becomes more volatile.

\begin{tabular}{|l|l|l|}
\hline Percentile & Avg. Atk. $(\epsilon=0.20)$ & Avg. Atk. $(\epsilon=0.99)$ \\
\hline $20^{t h}$ & 0.21688 & 0.648644 \\
$40^{t h}$ & 0.246331 & 0.677153 \\
$60^{t h}$ & 0.285942 & 0.717554 \\
$80^{t h}$ & 0.28811 & 0.715567 \\
$100^{t h}$ & 0.29328 & 0.722912 \\
\hline
\end{tabular}

Table 5: Average Atkinson index per Percentile, LANL Data.

The resulting Atkinson index values suggest that a system's age significantly affects the volatility of its failure behavior in failures causing both large and small impacts on downtime. However, there is a greater amount of inequality reported by the Atkinson $(\epsilon=0.99)$ index than the Atkinson $(\epsilon=0.20)$ index, which infers that larger failures have a more significant effect on the level of inequality contained in the data set than smaller failures. To illustrate this, consider System 13, which exhibits a single failure with an impact of 5.648 days. The Atkinson $(\epsilon=0.20)$ index for this system, which is associated with a parameter that lowered sensitivity to individual large failures, was only 0.4596139. The Atkinson $(\epsilon=0.99)$ index, which does not lower this sensitivity, was 0.9999994. Moving beyond the Atkinson index to a more accurate representation of 
this failure activity requires the derivation of a time-dependent inequality measure. The FI derived in this study generates a metric that measures time-dependent HPC system volatility, rendering percentile-based approaches such as in this section superfluous.

\subsection{DISCUSSION AND OBSERVATIONS}

Investigating the behavior of the 23 LANL systems we have observed that sixteen of the twenty three systems exhibit increasing ROCOF, while seven of the systems have decreasing ROCOF. However, increasing or decreasing ROCOF has little impact on the failure volatility of a given system. Systems with increasing ROCOF generated an $\mathrm{FI}(\epsilon$ $=0.99)$ of 0.632599 and systems with decreasing ROCOF generated an $\mathrm{FI}(\epsilon=0.99)$ of 0.659323 , which are not significantly different. Long periods of failure or non-failure behavior have a significant impact on the failure volatility of a given system. System 21, for example, contains a significant period of time without failure activity toward the end of its lifetime, resulting in an $\operatorname{FI}(\epsilon=0.99)$ of 0.821141 . This was the highest FI reported for any system. Likewise, System 20, which contains the most regular time intervals between each failure had an $\operatorname{FI}(\epsilon=0.99)$ of 0.3392873 , the lowest of any system.

It is important to reiterate that the FI is intended to be used as part of an ensemble of statistics representing failure activity and it is explicitly not a measure of good or bad behavior in its own right. Long stretches of non-failure activity have just as much impact on the resulting index as equally long stretches of continuous failure activity. The FI only captures these fluctuations and does not distinguish between either case. The indices utilized in this paper as means to analyze failures in an HPC environment Gini, Atkinson, GT and FI - should be considered complementary tools. Depending on a variety of factors impacting failure modes, for a given system one index may highlight some aspects of reliability that the other indices may not be sensitive to. To illustrate this we ask the reader to consider two sets of numerical values. Both sets may have the same mean, thus using only this metric in an evaluation fails to capture difference in the data sets. However, metrics such as standard deviation or the aforementioned Gini or Atkinson indices will be able to capture the differences. In addition the Atkinson index, unlike to Gini index, will illustrate where in the distribution the difference (inequality) lies - in the upper or the lower end of the data.

Let's examine System 19 in more detail. FI values are generated with parameters $\epsilon=0, \epsilon=0.20$ and $\epsilon=0.99$ as in Table 4. Further, a GT index is generated for each system using Equation (3). The GT index represents the system's ROCOF with respect to time. Increases in ROCOF generate positive GT index values. Likewise, decreases in ROCOF generate negative GT index values. Index values closer to 1 or -1 contain higher increases or decreases in ROCOF than index values closer to 0 .

\begin{tabular}{|l|l|l|l|l|l|l|}
\hline System & Percentile & Failures & $\begin{array}{l}\text { Downtime } \\
\text { (min.) }\end{array}$ & Gini & Atk(0.99) & Atk(0.20) \\
\hline 19 & $20^{t h}$ & 661 & 179209 & 0.7520437 & 0.6696452 & 0.2678224 \\
19 & $40^{t h}$ & 1298 & 278133 & 0.7269844 & 0.6355074 & 0.2520972 \\
19 & $60^{t h}$ & 1992 & 377358 & 0.7296928 & 0.640358 & 0.2476286 \\
19 & $80^{t h}$ & 2656 & 493133 & 0.7152188 & 0.6226461 & 0.2286325 \\
19 & $100^{t h}$ & 3284 & 681077 & 0.7409367 & 0.6561462 & 0.2670949 \\
\hline
\end{tabular}

Table 6: Inequality Indexes, System 19, LANL Data. 
The relationship between the Atkinson index, FI and ROCOF can be seen in the analysis of System 19. As shown in Table 6, the Atkinson index increases by $5-15 \%$ in the $20^{\text {th }}$ and $100^{\text {th }}$ percentiles due to large failures incurred in the early and late stages of the system's lifespan. These failures also manifest themselves when one graphs System 19 's FI with respect to time - small increases can be seen correlating to the initial group of large failures. The pair of substantial jumps on the left-hand side of Figure 1 are the result of the second group of failures.
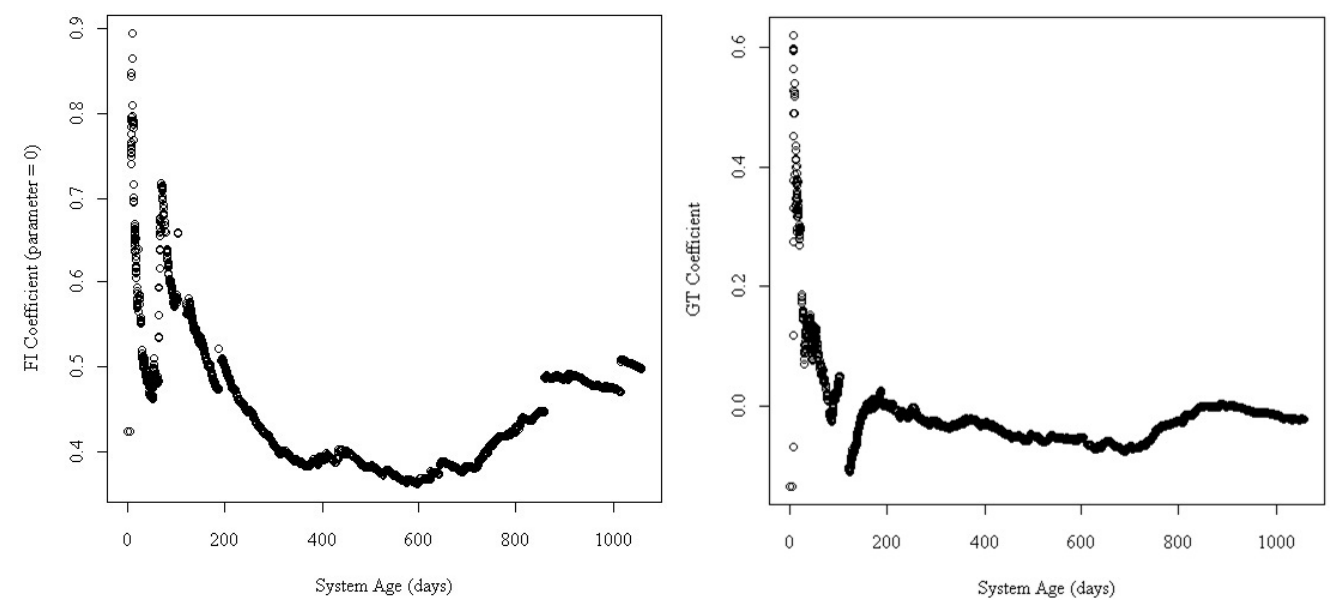

Figure 1: Lorenz Curves for Two Distributions with Varying Levels of Inequality. System 19 is depicted with the FI on y-axis computed for the $\epsilon=0$ (left) and GT coefficient (right). GT/ROCOF cannot capture the failures captured by FI and suggests a stable system.

However, neither set of failures manifest themselves in Figure 1 right-hand side, which graphs ROCOF with respect to time. System 19's ROCOF initially decreases then centers itself on 0 and remains there for the duration of the system's lifespan, illustrating a rather stable system. System 19 exhibits more interesting failure behavior than the other LANL systems and demonstrates the use and benefits of the each statistical measure proposed. We do not present analysis of the other systems.

Table 7 presents the results of the analysis when performed on all 23 systems, illustrating FI metrics for different values of $\epsilon$. Due to the lack of qualitative information released alongside the LANL data one can only speculate as to why, for example, system 20 has the lowest FI. The metrics proposed in this paper are tools to be used in the evaluation of given information, not to perform root cause analysis. We present potential applications of the FI in Section 5 . 


\begin{tabular}{|l|l|l|l|l|}
\hline System & FI $(\epsilon=0)$ & FI $(\epsilon=0.2)$ & FI $(\epsilon=0.99)$ & GT $(\mathrm{N}(\mathrm{T}))$ \\
\hline 2 & 0.3488647 & 0.3739617 & 0.5606060 & -0.2452266 \\
3 & 0.4762693 & 0.4928603 & 0.6238533 & -0.1400224 \\
4 & 0.5312802 & 0.5510522 & 0.6861621 & -0.1069729 \\
5 & 0.4517466 & 0.4683794 & 0.6250355 & -0.0570195 \\
6 & 0.4661334 & 0.4750499 & 0.5761766 & -0.0092863 \\
7 & 0.5590928 & 0.5754688 & 0.6686179 & -0.2554706 \\
8 & 0.5743319 & 0.5965732 & 0.7276079 & 0.1277645 \\
9 & 0.3801783 & 0.4195316 & 0.7901992 & 0.4110598 \\
10 & 0.4393362 & 0.4668898 & 0.6757968 & 0.3073982 \\
11 & 0.3228945 & 0.3503598 & 0.6125574 & 0.3016312 \\
12 & 0.3989725 & 0.4317884 & 0.6667910 & 0.3937295 \\
13 & 0.3546629 & 0.3805183 & 0.6209388 & 0.2985360 \\
14 & 0.3113004 & 0.3501405 & 0.7133446 & 0.1522754 \\
15 & 0.3203539 & 0.3445422 & 0.6678887 & -0.3574969 \\
16 & 0.5062350 & 0.5470675 & 0.7952932 & -0.1423502 \\
17 & 0.4431006 & 0.4693957 & 0.6992799 & -0.1168528 \\
18 & 0.4424562 & 0.4581639 & 0.5760466 & -0.0101157 \\
19 & 0.4978905 & 0.5130915 & 0.6412173 & -0.0219160 \\
20 & 0.2358755 & 0.2441450 & 0.3392873 & -0.3663878 \\
21 & 0.3521442 & 0.3752301 & 0.8211410 & -0.2562533 \\
22 & 0.4140757 & 0.4439510 & 0.6050619 & -0.1168699 \\
23 & 0.4729281 & 0.4977982 & 0.6634825 & -0.0031028 \\
24 & 0.3328124 & 0.3583177 & 0.5140770 & -0.3460315 \\
\hline
\end{tabular}

Table 7: Failure Index for the 23 systems, LANL Data.

\section{CONCLUSIONS AND FUTURE APPLICATIONS}

In this study we introduced a set of statistics - specifically the derivation of a new failure index - that can be used in the evaluation of High Performance Computing reliability with a focus on how each of these measures complement one another when performing a thorough analysis.

The FI is applicable to any multi-tenant distributed computing environment including HPC and Big Data analytic platforms. In HPC, job schedulers such as SLURM, PBS, or LSF can leverage the FI to provide application- and user-level perspective on resource utilization and operational risk. Users and applications exhibiting higher FI values relative to others concurrently using the shared hardware resource can be provisioned additional fault tolerance capabilities such as more frequent checkpointing. Multi-tenant distributed data analytic platforms such as Hadoop and Spark can utilize the FI in a similar fashion. Here resource managers (such as YARN) can apply user- and applicationlevel FI values to handle faulty and stable applications in a specified manner. This can include turning on data replication for data sets underpinning fault-likely applications or containerizing particularly volatile logic using tools such as Docker. Likewise stable analytics with low FI values can allow for the removal of data replication, increasing performance and storage capacity. In addition to platform administrators, users of both HPC and Big Data systems can leverage the FI to review the impact of their source code on the stability of the underlying resource (and, subsequently, concurrent users). Particularly in relatively new technologies such as Spark and Hadoop, it is quite easy 
for a user to unknowingly bring a system to its knees by making more algorithm implementation decisions [9]. Quantifying the faulty behavior of an application using metrics such as FI can help authors better understand their applications and make healthier implementation choices. It is important to note that the FI is intended for batch-oriented processing systems. If a given user's analytic workflow consists of exploratory analysis (through, for example, the Spark REPL) wherein one makes execution decisions in real time, the FI cannot work as intended. This metric, like others derived through statistical inference, models a corpus of past data to draw conclusions about the present. In HPC and Big Data processing, this equates to resource allocation schemes leveraging batches and queues and a history of successful and unsuccessful jobs.

By combining the Failure Index and other econometric-based statistics with an existing ensemble of analytic methods in monitoring and studying HPC failures, one can gain a better understanding of these systems' behavior.

\section{Acknowledgments}

The authors gratefully acknowledge financial support from the National Science Foundation under award number \#EPS-1003897 as well as the EU research project FP7-ICTFET-Proactive award number \#612146 (PLASWIRES) and ANCS PN-II-PT-PCCA2011-3.2-1710 (RemoteForest). We would also like to thank the anonymous reviewers who have helped improve significantly the quality of the manuscript.

\section{References}

[1] Atkinson A. B., 1970. On the measurement of inequality. Journal of Economic Theory 2(3), 244 $-263$.

[2] Atkinson, A. B., Bourguignon, F. (Eds.) 2000. Handbook of income distribution (Vol. 1). Elsevier, North Holland. 938 pages.

[3] Barbour A. D., Mansson M., 2002. Compound Poisson process approximation. Annals of Probability 30(3): $1492-1537$.

[4] Burkimsher A., Bate I., Thomas M., 1991. Scheduling HPC Workflows for Responsiveness and Fairness with Networking Delays and Inaccurate Estimates of Execution Times, Euro-Par 2013 Parallel Processing series Lecture Notes in Computer Science 8097: 126 - 137.

[5] Cappello F., Geist A., Gropp B., et al., 2013. Toward Exascale Resilience. International Journal of High Performance Computing Applications 23(4): $374-388$.

[6] Chandler C., 2012. A failure index for high performance computing applications. PhD Dissertation, Louisiana Tech University, 202 pages ISBN: 978-1-267-25534-1.

[7] Chandler C., DeBardeleben N., Leangsuksun C., 2010. Resilience Analysis of High Performance Computing Applications via Bayesian Pattern Recognition. The National Workshop on HPC Resilience, August 2010.

[8] Dongarra J., Beckman P., Aerts P., et al., 2009. The International Exascale Software Project: A Call to Cooperative Action by the Global High-Performance Community. International Journal of High Performance Computing Applications 23(4): 309 - 322.

[9] Doulkeridis, C., Norvag, K., 2013. A Survey of Large-Scale Analytical Query Processing in MapReduce. The VLDB Journal 23(3): $355-380$.

[10] El-Sayed N., Schroeder B., 2013. Reading between the lines of failure logs: Understanding how HPC systems fail, DSN '13 Proceedings of the 2013 43rd Annual IEEE/IFIP International Conference on Dependable Systems and Networks (DSN), $1-12$.

[11] Gottumukkala N.R., Leangsuksun C., Nassar R., et al., 2007. Reliability-aware resource allocation in HPC systems. Proceedings of the IEEE International Conference on Cluster Computing.

[12] He X., Ou L., Engelmann C., Chen X., Scott S., 2009. Symmetric Active/Active Metadata Service for High Availability Parallel File Systems, Journal of Parallel and Distributed Computing, 69(12): $961-973$ 
[13] Jones-Wyatt, E., Domerçant, J.C., Mavris, D.N., 2013. A reliability-based measurement of interoperability for systems of systems. In Systems Conference (SysCon), 2013 IEEE International (pp. 408-413). IEEE.

[14] Kaminskiy M.P., Krivtsov V., 2010. A Gini-Type Index for Aging/Rejuvenating Objects. In Mathematical and Statistical Models and Methods in Reliability Springer, Birkhäuser Boston ISBN: 978-0-8176-4970-8

[15] Kavulya, S., Tan, J., Gandhi, R., Narasimhan, P., 2010. An analysis of traces from a production mapreduce cluster. In Cluster, Cloud and Grid Computing (CCGrid), 201010 th IEEE/ACM International Conference on (pp. $94-103)$. IEEE.

[16] Lima M/ A. de Q.V, Maciel P. R.M., Silva B., Guimarães A. P., 2014. Performability evaluation of emergency call center, Performance Evaluation 80: $27-42$.

[17] Liu T., 2004. High Availability and Performance Linux Cluster. Master's Thesis, Louisiana Tech University, Ruston, LA, USA.

[18] Lorenz M.O., 1905. Methods of measuring the concentration of wealth. Publications of the American Statistical Association 9(70): 209 - 219.

[19] Nakka, N., Agrawal, A., Choudhary, A. 2011. Predicting node failure in high performance computing systems from failure and usage logs. In Parallel and Distributed Processing Workshops and Phd Forum (IPDPSW), 2011 IEEE International Symposium on (pp. 1557 - 1566). IEEE.

[20] Pal A., Agarwala A., Raha S., Bhattacharya B., 2014. Performance metrics in a hybrid MPIOpenMP based molecular dynamics simulation with short-range interactions. Journal of Parallel And Distributed Computing 74(3): $2203-2214$

[21] Pesonen E., 1968. On definition of compound Poisson processes. Scandinavian Actuarial Journal 1968(3-4): $156-157$.

[22] Sarabia J.M., Azpitarte F., 2012. On the relationship between objective and subjective inequality indices and the natural rate of subjective inequality. ECINEQ $2012-248$

[23] Schroeder B., Gibson G.A., 2006. A Large-Scale Study of Failures in High Performance Computing Systems. In Proceedings of the International Conference on Dependable Systems and Networks (DSN2006).

[24] Strîmbu B.M., Innes J.L., 2012. Framework for assessing the impact of human activities on the environment: the impact of forest harvesting and petroleum drilling on habitat of moose (Alces alces) and marten (Martens americana). Biodiversity and Conservation 21(4): $733-755$.

[25] Strîmbu B.M., Hickey, G.M., Strîmbu V.G., Innes J.L., 2009. On the use of statistical tests with non-normaly distributed data in landscape change detection. Forest Science 55-1: $72-83$.

[26] Păun M., 2006. Measuring Inequality: Statistical Inferential Theory with Applications. PhD Dissertation, Louisiana Tech University, 142 pages, ISBN: 978-0-542-70044-6. 